\title{
Association of prematurity with the development of infantile hypertrophic pyloric stenosis
}

\author{
Christopher M. Stark', Philip L. Rogers ${ }^{2}$, Matthew D. Eberly ${ }^{1,2}$ and Cade M. Nylund ${ }^{1,2}$
}

BACKGROUND: Infantile hypertrophic pyloric stenosis (IHPS) has several known risk factors. The association between prematurity and IHPS and the timeline of presentation are poorly defined. Our aim was to evaluate the associations between IHPS and prematurity.

METHODS: We performed a retrospective cohort study of 1,074,236 children born between June 2001 and April 2012 in the US Military Health System. IHPS cases and gestational ages (GA) were identified using billing codes. Additional risk factors for IHPS were controlled for in a multivariable logistic regression model.

RESULTS: The incidence of IHPS was 2.99 per 1,000 in preterm infants and 2.25 per 1,000 in full term (relative risk $(R R)=1.33$, 95\% confidence interval (Cl) 1.16-1.54). The adjusted odds ratio for prematurity was 1.26 (95\% Cl 1.08-1.46). The median (interquartile range (IQR)) chronological age at presentation was $40 \mathrm{~d}(30-56)$ in preterm infants vs. $33 \mathrm{~d}(26-45)$ in full term $(P<0.001)$. Median postmenstrual age at presentation was 42 wk in preterm infants (40-42) vs. 45 wk (44-46) in full term $(P<0.001)$.

CONCLUSION: Prematurity is associated with IHPS. Premature infants develop IHPS at a later chronological age, but earlier postmenstrual age, than term infants. Providers should have an increased concern for IHPS development in premature infants.

nfantile hypertrophic pyloric stenosis (IHPS) is a common cause of gastric outlet obstruction in young infants. IHPS is a significant source of infant morbidity and, left untreated, can cause severe dehydration and life-threatening metabolic alkalosis. IHPS is now recognized earlier in its clinical course and is managed successfully with surgical pyloromyotomy (1-4). There are several established prenatal and perinatal risk factors for IHPS, including macrolide antibiotic use, bottle-feeding, male sex, family history, and birth order (5-8). The etiology of IHPS remains unclear, but current research suggests that it is caused by an interplay of environmental and genetic factors (9). Retrospective studies and post-hoc analysis with large populations continue to add important information about the potential causes and clinical course of IHPS.
The rate of preterm birth in the United States, defined as delivery at $<37 \mathrm{wk}$ gestation, was around $11.7 \%$ in 2011 (10). The unique risks and clinical course of diseases in preterm populations are often underappreciated. The understanding of a relationship between IHPS and prematurity has evolved. Previous literature suggested that preterm infants rarely present with IHPS $(11,12)$. Several recent epidemiological studies have found variable evidence that prematurity might be associated with increased risk of developing IHPS, although this association remains unclear $(7,8,13,14)$.

Full-term infants with IHPS typically present in the first $2-7 \mathrm{wk}$ of life (7). The presentation age of preterm infants that develop IHPS is defined less clearly. Some studies suggest that preterm infants may present with IHPS symptoms at a later chronological age than term infants, but the differences between presentation times in full-term and preterm infants are poorly characterized $(7,8,11,13-15)$. Using data from the Military Health System database, this study aims to examine the associations between prematurity and IHPS, and to investigate both gestational age (GA) and chronological age of IHPS among premature infants.

\section{RESULTS}

The characteristics of preterm and full-term infants that met inclusion criteria are presented in Table 1 . Of the 1,074,236 children born during the 11-y study period, 69,517 (6.5\%) were preterm $(<37 \mathrm{wk}$ GA). Preterm infants were significantly more likely to be males, be a part of a multiple gestation birth, and have exposure to macrolide antibiotics $(P<0.001)$.

\section{Pyloric Stenosis Incidence}

The rates and relative risks for IHPS are outlined in Table 2. A total of 2,466 infants within the study population had IHPS, with an overall incidence of 2.29 per 1,000. As expected, the relative risk of IHPS was increased significantly in male sex, firstborn status, multiple gestation births, and infants with recorded macrolide exposure. Full-term infants $(\geq 37 \mathrm{wk}$ GA) had an IHPS incidence of 2.25 per 1,000. There were a total of 208 cases of IHPS in premature infants, with an incidence of 2.99 per 1,000 and overall relative risk of 1.33 (95\% CI 1.16-1.54). Compared to full-term infants, preterm infants

'Department of Pediatrics, F. Edward Hébert School of Medicine, Uniformed Services University of the Health Sciences, Bethesda, Maryland; ${ }^{2}$ Department of Pediatrics, Walter Reed National Military Medical Center, Bethesda, Maryland. Correspondence: Christopher M. Stark (christopher.stark@nccpeds.com)

Received 7 February 2015; accepted 26 March 2015; advance online publication 10 June 2015. doi:10.1038/pr.2015.92 
stratified by GA groups had statistically significant increases in IHPS incidence in the 33-34 wk (RR 1.51, 95\% CI 1.16-1.97) and 35-36 wk (RR 1.34, 95\% CI 1.11-1.62) cohorts. Premature infants in the individual 23-32 wk GA groups did not have a significant increase risk of IHPS; however, the numbers within these individual GA groups were small.

On univariate and multivariable analysis, prematurity, male sex, macrolide exposure, multiple gestation births, and firstborn status were significant independent predictors of IHPS risk (Table 3). Of note, there was no significant change in the rate of pyloric stenosis over the study period $(0.54 \%$ average annual decrease, $P=0.25$ ) and $2.48 \%$ average annual increase in the rate of prematurity $(P<0.001)$ over the study period (data not shown).

\section{Presentation Ages}

Gestational, chronological, and postmenstrual ages at presentation with IHPS were compared between preterm and term

Table 1. Characteristics of preterm and full-term infants

\begin{tabular}{lcccc}
\hline & $\begin{array}{c}\text { Preterm } \\
(<37 \mathrm{wk})\end{array}$ & $\begin{array}{c}\text { Full term } \\
(\geq 37 \mathrm{wk})\end{array}$ & Pvalue & Total \\
\hline Number & 69,517 & $10,047,19$ & - & $10,742,36$ \\
Male $^{\mathrm{a}}$ & 37,081 & 516,058 & $<0.001$ & 553,139 \\
& $(53.34 \%)$ & $(51.36 \%)$ & & $(51.49 \%)$ \\
Firstborn $^{\mathrm{a}}$ & 29,230 & 455,379 & $<0.001$ & 486,887 \\
& $(42.95 \%)$ & $(45.55 \%)$ & & $(45.32 \%)$ \\
Multiple & 14,822 & 13,916 & $<0.001$ & 28,738 \\
gestations & $(21.32 \%)$ & $(1.39 \%)$ & & $(2.68 \%)$ \\
Macrolide & 545 & 6,364 & $<0.001$ & 6,909 \\
exposure & $(0.78 \%)$ & $(0.63 \%)$ & & $(0.64 \%)$ \\
\hline
\end{tabular}

aMissing data included 34 infants with unknown sex and 12,514 with unknown birth order status. Percentages are calculated based on nonmissing data within category.
Table 3. Univariate and multivariable logistic regression results for infantile hypertrophic pyloric stenosis

\begin{tabular}{lcc}
\hline Risk factor & $\begin{array}{c}\text { Univariate } \\
\text { OR }(95 \% \mathrm{Cl})\end{array}$ & $\begin{array}{c}\text { Multivariate } \\
\text { OR }(95 \% \mathrm{Cl})\end{array}$ \\
\hline Prematurity $(<37 \mathrm{wk})$ & $1.33(1.16-1.54)$ & $1.26(1.08-1.46)$ \\
Male sex & $4.49(4.04-4.98)$ & $4.48(4.04-4.97)$ \\
Macrolide exposure & $2.58(1.07-2.35)$ & $1.52(1.02-2.25)$ \\
Multiple gestations & $1.38(1.12-1.70)$ & $1.32(1.06-1.67)$ \\
Firstborn status & $1.22(1.12-1.32)$ & $1.23(1.13-1.33)$ \\
\hline
\end{tabular}

The complete multivariable analysis also included year. A two-way interaction between year and infantile hypertrophic pyloric stenosis rates was controlled for in the final model.

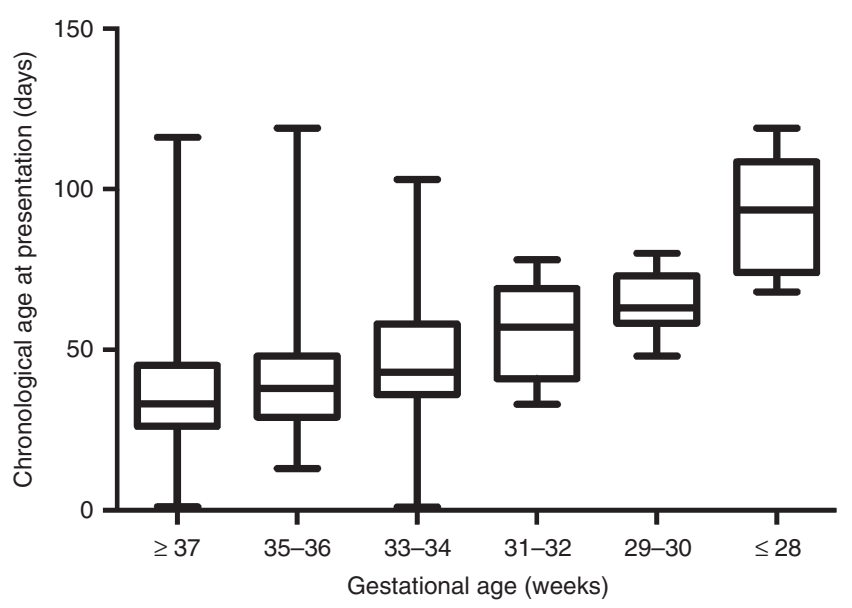

Figure 1. Chronological age at presentation with infantile hypertrophic pyloric stenosis. The chronological age at presentation was found to generally increase with lower estimated gestational age infants.

Table 2. Rates and relative risks for infantile hypertrophic pyloric stenosis

\begin{tabular}{|c|c|c|c|c|}
\hline & Total in group & Number with IHPS & Rate per 1000 & Relative Risk (95\% Cl) \\
\hline All infants & $10,742,36$ & 2,466 & 2.29 & - \\
\hline Male $^{a}$ & $553,139(51.5 \%)$ & $2,037(82.7 \%)$ & 3.70 & $4.50(4.05-4.99)$ \\
\hline Multiple gestations & $28,648(2.67 \%)$ & $90(3.7 \%)$ & 3.14 & $1.38(1.12-1.70)$ \\
\hline Macrolide exposure & $6,909(0.64 \%)$ & $25(1.01 \%)$ & 3.62 & $1.58(1.07-2.35)$ \\
\hline \multicolumn{5}{|l|}{ Gestational age } \\
\hline Full-term infants ( $\geq 37 \mathrm{wk}$ ) & $10,047,19(93.53 \%)$ & $2,258(91.57 \%)$ & 2.25 & Reference \\
\hline Preterm infants ( $<37 w k)$ & $69,517(6.47 \%)$ & $208(8.43 \%)$ & 2.99 & $1.33(1.16-1.54)$ \\
\hline $23 w k$ & 214 & 0 & - & - \\
\hline $24 w k$ & 427 & 1 & 2.34 & $1.02(0.14-7.26)$ \\
\hline $25-26 w k$ & 1,424 & 2 & 1.40 & $0.61(0.15-2.45)$ \\
\hline $33-34$ wk & 16,591 & 57 & 3.44 & $1.51(1.16-1.97)$ \\
\hline $35-36 w k$ & 38,498 & 117 & 3.04 & $1.34(1.11-1.62)$ \\
\hline
\end{tabular}

aMissing data included 34 infants with unknown sex and 12,514 with unknown birth order status. Percentages are calculated based on nonmissing data within category. 


\section{Articles $\mid$ Pyloric stenosis in premature infants}

infants. Chronological age at presentation was found generally to increase as GA decreased (Figure 1). Preterm birth was associated with longer interval from birth to presentation with IHPS: median (IQR) days of $40(30-56)$ vs. $33(26-45)$ in full-term infants, $P<0.001$ (Figure 2). Premature infants were found to present at an earlier median estimated postmenstrual age: $42 \mathrm{wk}(40-42)$ vs. $45 \mathrm{wk}(44-46)$ in full-term infants, $P<0.001$ (Figure 3).

\section{DISCUSSION}

Rates of premature birth are increasing globally and overall survival of preterm infants continues to improve. Analyses of large, diverse populations of preterm infants are necessary to evaluate the disease risk within this growing cohort. There are no previous studies assessing IHPS rates in a large cohort of preterm infants in the United States. In this study, we compared the rates and presentation ages of preterm and term infants with IHPS, and found that preterm birth was associated with IHPS. Additionally, we found that preterm infants

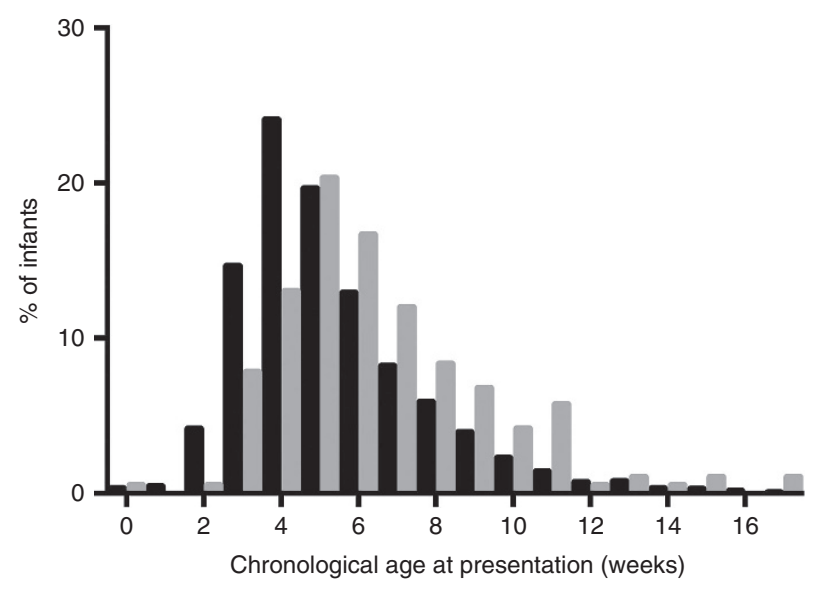

Figure 2. Distribution of chronological age at time of infantile hypertrophic pyloric stenosis in full-term (black bars) and preterm (gray bars) infants. The median chronological age at presentation was significantly later in preterm infants compared to full-term infants $(P<0.001)$.

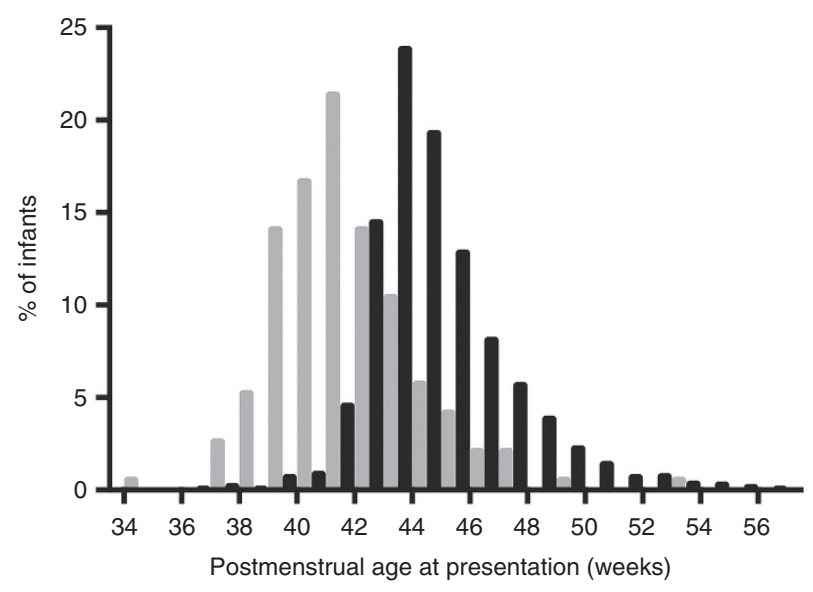

Figure 3. Distribution of postmenstrual age at time of infantile hypertrophic pyloric stenosis in full-term (black bars) and preterm (gray bars) infants. The median postmenstrual age at presentation with was significantly earlier in preterm infants compared to full-term infants $(P<0.001)$. present at a later chronological age, but earlier postmenstrual age, than term infants.

The development of IHPS in preterm infants has been described previously as a rare entity $(11,12)$. Previous studies have assessed rates of IHPS in US populations, but have not observed increased rates of IHPS in premature infants. One previous study of 1,963 California infants with a mean GA of $39.5 \mathrm{wk}$ found no increased rate of IHPS in preterm infants, but predicted that preterm infants might see an increase in IHPS rates as the number of infants that survive past the first weeks of life increases (15). It is unknown whether the previous understanding that IHPS was rare in prematurity was due to small study population sizes, low preterm infant survival rates, missed diagnosis, or if the present findings represent a new shift in disease epidemiology. Few studies have appropriately large sample sizes to assess IHPS risk in premature infants. Studies of Australian and Scandinavian infants have found increased rates of IHPS in preterm infants $(14,16)$. The Australian hospital study found that up to $19 \%$ of cases of IHPS occurred in infants born prematurely, while the population-wide Scandinavian study found that prematurity was a risk factor for IHPS (OR 2.54, 95\% CI 2.06-3.14). Ours is the first US population study to recognize a significant increase in IHPS rates among preterm infants.

The present study supports previous findings that male sex and firstborn status are associated with increased risk for IHPS. We also found that multiple gestations is a risk factor for pyloric stenosis, an association that has been previously described in a large Danish cohort study (17). This study reported that the risk of IHPS was much higher among monozygotic than dizygotic twins. The association of multiple gestations with IHPS in our study likely represents familial aggregation, but additional data on zygosity was not available. Macrolide exposure also has been found to be associated with IHPS $(18,19)$. One recent study found that exposure to different macrolides in the perinatal period can increase the risk of developing IHPS (20). Preterm infants may have increased macrolide exposure for infection prophylaxis or medical management of delayed gastric emptying. In the present study, after adjusting for these prematurity-associated risk factors, premature infants were still found to have a significantly increased risk of IHPS.

IHPS typically develops at $2-7 \mathrm{wk}$ chronological age in full-term infants, but several previous studies have observed that preterm infants appear to present at a later chronological age $(14,15)$. The current study confirms these findings and also found that preterm infants present at an earlier estimated postmenstrual age than full-term infants. It has been suggested previously that a certain gastrointestinal maturity threshold must be reached for IHPS to develop. Preterm infants, according to postmenstrual age, appear to meet this gut maturation threshold earlier than term infants. Interestingly, preterm infants do not experience migrating motor complexes until $32 \mathrm{wk}$ of gestation, the point when motilin receptors become functionally present (21). Relative immaturity of the gastrointestinal tract may preclude the development of IHPS in very premature infants, but a larger 
sample size is needed to evaluate this population. Further analysis of larger premature infant cohorts is necessary to determine the clinical significance of postmenstrual age in the development of IHPS.

Full-term infants with IHPS typically have projectile vomiting, a palpable olive-like mass in the right upper quadrant, and characteristic ultrasound findings (14). Preterm infants often lack these characteristic clinical findings $(22,23)$. IHPS may be under-recognized because of its atypical clinical presentation and time course of disease (14). Additionally, preterm infants have a lower body weight at admission, require more complex diagnostic modalities, and have higher postoperative complication rates than term infants (8).

Established IHPS risk factors, including male sex, firstborn status, multiple gestations, and outpatient macrolide exposure were controlled in our statistical analysis. Preterm infants often require extended neonatal intensive care unit (NICU) stays, which may lead to additional risk factor exposure. Bottle-feeding formula, recently recognized to increase risk of IHPS, may be more common in a NICU setting (6). Preterm infants may require prolonged prostaglandin therapy to maintain ductus arteriosus patency. Increased physiologic and therapeutic prostaglandin levels have been associated previously with reactive gastropathy and gastric outlet obstruction (24-26). Maternal smoking, a known risk factor for premature birth, also has been associated with increased risk of IHPS (16). Bottle-feeding status, inpatient medication exposures, and maternal smoking data were unavailable for the present study. Of note, previous studies of preterm infants in military families have reported low rates of maternal smoking (27). Additionally, military families have access to low cost or free healthcare, higher rates of maternal education, and lower selfreported fetal exposure to alcohol or drug use. These findings are believed to contribute to the overall lower rate of prematurity observed in the present study. Although the prematurity rate was lower in our study population, the identified association between prematurity and IHPS would still be valid.

There are several strengths to this study. Utilizing TRICARE beneficiaries within the Military Health System offers a large sample of demographically and geographically diverse newborns from military and civilian facilities. The large size of our population facilitates identifying an association of a relatively uncommon condition of IHPS. Selection bias is minimized because TRICARE beneficiaries have universal healthcare for hospitalization and emergent procedures, maximizing captured events. The database used in this study included outpatient and hospital discharge ICD-9-CM diagnostic and procedural codes. As in all studies utilizing billing coding, misclassification bias is possible. We attempted to limit misclassification bias by restricting the definition of IHPS to having both diagnostic codes and procedural codes for pyloric stenosis and pyloromyotomy. Diagnostic codes for GA are based on standard of care GA estimates, including prenatal ultrasound and LMP calculation. These methods of estimating GA are very reliable, but technical errors and LMP miscalculations may still occur and we acknowledge this limitation in the study. Additionally, we recognize that our groupings of gestation age are broad, and postmenstrual age as presented represents only an estimate.

In conclusion, this study provides additional evidence that prematurity is an independent risk factor for IHPS. Additionally, preterm infants typically present at a later chronological age than full-term infants. Preterm infants may have a less specific clinical presentation and have been shown to have more negative clinical outcomes; therefore, an increased index of suspicion is necessary to prevent delayed diagnosis. Further focused research must be done to characterize possible environmental and intrinsic risk factors for the development of IHPS in the premature population.

\section{METHODS}

\section{Study Cohort}

The protocol for this study was reviewed and approved by the Uniformed Services University of the Health Sciences Institutional Review Board. Consent for use of personal data was not obtained because all personally identifiable information was deidentified from the data source and informed consent was not required. A retrospective cohort study of children born between June 2001 and April 2012 was performed, as reported in the TRICARE Management Activity (TMA) Military Health System (MHS) database. The MHS consists of each of the components within the Office of the Assistant Secretary of Defense for Health Affairs, which includes the medical departments of the Army, Navy, Air Force, and providers at civilian facilities. The TMA oversees health care delivery for US uniformed services members and their families. The MHS database includes all outpatient and inpatient billing records for all eligible military dependents in both military and civilian facilities. The MHS database was queried for all children eligible for TRICARE healthcare starting at birth. Children were included in the cohort if they had a record of birth and were in the system up to $120 \mathrm{~d}$ of life.

\section{Variable Definitions}

Prematurity by GA groups was determined from the infant's birth record using International Classifications of Diseases, Ninth Revision, Clinical Modification (ICD-9-CM) codes, to include 765.2 series values ranging from 765.2123 completed weeks of gestation through 765.2937 or more completed weeks of gestation. The age of diagnosis of IHPS was determined from the admission date that included pyloromyotomy, which is the corrective surgical procedure for IHPS. An estimated postmenstrual age was determined by adding the estimated weeks of gestation to the chronological age at diagnosis and treatment for IHPS. Cases of IHPS were identified using specific diagnostic ICD-9-CM codes, to include 750.5 congenital hypertrophic pyloric stenosis, 537.0 acquired hypertrophic pyloric stenosis, or 537.81 pylorospasm. In addition to having at least one of the above codes, children classified as IHPS also were required to have a Current Procedural Terminology code of 43520 , or a ICD-9-CM procedure code of 43.3 (pyloromyotomy). The combination of a diagnostic code and procedure code was used to minimize misclassification bias. Multiple gestations were defined using ICD-9-CM codes V31x and V34x. A child was classified as firstborn if he or she was the first, eligible, dependent child of the uniformed member on record. Macrolide exposure was determined from outpatient prescription records and included any history of erythromycin, azithromycin, or clarthromycin dispensed and billed. No inpatient macrolide prescribing data were available.

\section{Statistical Analysis}

Univariate analyses were conducted to investigate normality of the data. We summarized normally distributed data with means and SD and skewed data with median and interquartile ranges. The $\chi^{2}$ test was used to test differences of categorical variables. Wilcoxon Two-Sample test was used to compare continuous data between groups, including the differences between premature infants and term infant chronological age at time of the development of IHPS. Additional risk factors for 


\section{Articles | Pyloric stenosis in premature infants}

IHPS, including male sex, multiple gestation births, firstborn status, macrolide exposure, and year, were identified and controlled for in a multivariable logistic regression model. Statistical comparisons were made between GA and age at presentation of infants with IHPS. Twoway interactions between the independent variables were evaluated, and an interaction between yearly IHPS incidence and prematurity rates was accounted for in the final model. Statistical analyses were performed using SAS version 9.3 (SAS Institute, Cary, NC) and all statistical tests were performed at a significance level of $\alpha=0.05$. This study was reviewed and approved by the responsible institutional review board. The views expressed in this article are those of the authors and do not reflect the official policy or position of the United States Air Force, United States Army, Department of Defense, or the U.S. Government.

\section{STATEMENT OF FINANCIAL SUPPORT}

No funding was secured for this study.

Disclosure: The authors have no financial relationships relevant to products discussed in this study or potential conflicts of interest to disclose.

\section{REFERENCES}

1. Ein SH, Masiakos PT, Ein A. The ins and outs of pyloromyotomy: what we have learned in 35 years. Pediatr Surg Int 2014;30:467-80.

2. Taylor ND, Cass DT, Holland AJ. Infantile hypertrophic pyloric stenosis: has anything changed? J Paediatr Child Health 2013;49:33-7.

3. Papadakis K, Chen EA, Luks FI, Lessin MS, Wesselhoeft CW Jr, DeLuca FG. The changing presentation of pyloric stenosis. Am J Emerg Med 1999;17:67-9.

4. Poon TS, Zhang AL, Cartmill T, Cass DT. Changing patterns of diagnosis and treatment of infantile hypertrophic pyloric stenosis: a clinical audit of 303 patients. J Pediatr Surg 1996;31:1611-5.

5. Lund M, Pasternak B, Davidsen RB, et al. Use of macrolides in mother and child and risk of infantile hypertrophic pyloric stenosis: nationwide cohort study. BMJ 2014;348:g1908.

6. Krogh C, Biggar RJ, Fischer TK, Lindholm M, Wohlfahrt J, Melbye M. Bottle-feeding and the Risk of Pyloric Stenosis. Pediatrics 2012;130:e943-9.

7. Krogh C, Gørtz S, Wohlfahrt J, Biggar RJ, Melbye M, Fischer TK. Pre- and perinatal risk factors for pyloric stenosis and their influence on the male predominance. Am J Epidemiol 2012;176:24-31.

8. Huang IF, Tiao MM, Chiou CC, Shih HH, Hu HH, Ruiz JP. Infantile hypertrophic pyloric stenosis before 3 weeks of age in infants and preterm babies. Pediatr Int 2011;53:18-23.

9. Panteli C. New insights into the pathogenesis of infantile pyloric stenosis. Pediatr Surg Int 2009;25:1043-52.

10. Hamilton BE, Hoyert DL, Martin JA, Strobino DM, Guyer B. Annual summary of vital statistics: 2010-2011. Pediatrics 2013;131:548-58.
11. Adelstein P, Fedrick J. Pyloric stenosis in the Oxford Record Linkage Study area. J Med Genet 1976;13:439-48.

12. Katumba-Lunyenya J, Misra I, Chawda N, Erumbala G. Can you be too premature to develop pyloric stenosis? BMJ Case Rep 2012;2012:.

13. Rasmussen L, Green A, Hansen LP. The epidemiology of infantile hypertrophic pyloric stenosis in a Danish population, 1950-84. Int J Epidemiol 1989;18:413-7.

14. Gotley LM, Blanch A, Kimble R, Frawley K, Acworth JP. Pyloric stenosis: a retrospective study of an Australian population. Emerg Med Australas 2009;21:407-13.

15. Schechter R, Torfs CP, Bateson TF. The epidemiology of infantile hypertrophic pyloric stenosis. Paediatr Perinat Epidemiol 1997;11:407-27.

16. Svenningsson A, Svensson T, Akre O, Nordenskjöld A. Maternal and pregnancy characteristics and risk of infantile hypertrophic pyloric stenosis. J Pediatr Surg 2014;49:1226-31.

17. Krogh C, Fischer TK, Skotte L, et al. Familial aggregation and heritability of pyloric stenosis. JAMA 2010;303:2393-9.

18. Centers for Disease Control and Prevention. Hypertrophic pyloric stenosis in infants following pertussis prophylaxis with erythromycin--Knoxville, Tennessee, 1999. MMWR Morb Mortal Wkly Rep 1999;48:1117-20.

19. Honein MA, Paulozzi LJ, Himelright IM, et al. Infantile hypertrophic pyloric stenosis after pertussis prophylaxis with erythromcyin: a case review and cohort study. Lancet 1999;354:2101-5.

20. Eberly MD, Eide MB, Thompson JL, Nylund CM. Azithromycin in early infancy and pyloric stenosis. Pediatrics 2015;135:483-8.

21. Jadcherla SR, Klee G, Berseth CL. Regulation of migrating motor complexes by motilin and pancreatic polypeptide in human infants. Pediatr Res 1997;42:365-9.

22. Henderson JL, Brown JJ, Taylor WC. Clinical observations on pyloric stenosis in premature infants. Arch Dis Child 1952;27:173-8.

23. Hsu P, Klimek J, Nanan R. Infantile hypertrophic pyloric stenosis: does size really matter? J Paediatr Child Health 2014;50:827-8.

24. Perme T, Mali S, Vidmar I, et al. Prolonged prostaglandin E1 therapy in a neonate with pulmonary atresia and ventricular septal defect and the development of antral foveolar hyperplasia and hypertrophic pyloric stenosis. Ups J Med Sci 2013;118:138-42.

25. Lacher M, Schneider K, Dalla Pozza R, Schweinitz DV. Gastric outlet obstruction after long-term prostaglandin administration mimicking hypertrophic pyloric stenosis. Eur J Pediatr Surg 2007;17:362-4.

26. Shinohara K, Shimizu T, Igarashi J, Yamashiro Y, Miyano T. Correlation of prostaglandin E2 production and gastric acid secretion in infants with hypertrophic pyloric stenosis. J Pediatr Surg 1998;33:1483-5.

27. Holston A, Stokes T, Olsen C, et al. Novel noninvasive anthropometric measure in preterm and full-term infants: normative values for waist circumference:length ratio at birth. Pediatr Res 2013;74:299-306. 